\title{
Autopsie d'un fiasco organisationnel. Les applications du Paradoxe d'Abilène à une entreprise familiale
}

\author{
Gérard Ouimet, Ph.D. ${ }^{1}$ \\ HEC Montréal
}

\section{Introduction}

Le Paradoxe d'Abilène tire son nom d'une ville du centre du Texas où le chercheur Jerry Harvey ${ }^{2} a$ vécu une expérience fort singulière lors d'un dimanche après-midi de juillet 1971. Cet après-midi-là se tenait à Coleman, une petite ville située à 85 kilomètres d'Abilène, une réunion familiale chez les beauxparents de Jerry Harvey. Une chaleur torride enveloppait toute la région. Le mercure indiquait 40 degrés Celsius. Jerry Harvey, son épouse et ses beaux-

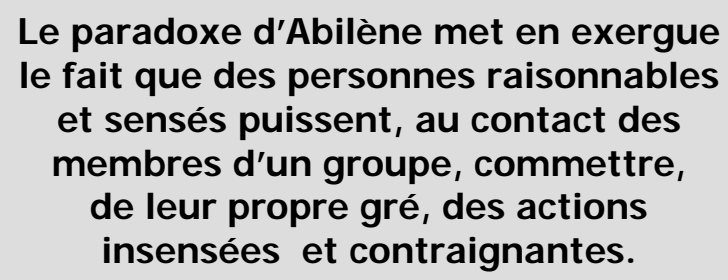

Dans une chaleur suffocante, nos intrépides voyageurs affrontèrent une véritable tempête de sable. Fin comme du talc, la poussière du désert emprunta les prises d'air du véhicule pour se répandre dans tout l'habitacle. Au contact avec la sueur des passagers, la poussière forma une croûte grisâtre qui les enveloppa de la tête au pied. Ils prirent rapidement l'allure des personnages tout enfarinés de la comedia dellarte. Après cette éprouvante traversée du désert, nos quatre aventuriers des dunes eurent droit à un parents étaient tapis à l'ombre, dans le jardin, tout près d'un vaillant ventilateur qui ronronnait allègrement. Tout en sirotant une limonade bien froide, les quatre victimes de la canicule jouaient paisiblement aux dominos. Soudainement, le beau-père de Jerry proposa d'aller souper dans un casse-croûte à Abilène. Fort peu emballé par l'idée de s'engouffrer, sous un soleil de plomb, dans une Buick 1958 dépourvue de climatisation, Jerry resta sans mot dire. C'est alors que son épouse trouva littéralement géniale la suggestion de son paternel. Ne voulant nullement assumer le rôle de rabat-joie, Jerry, quoique contrarié, acquiesça au projet et demanda à sa belle-mère ce qu'elle en pensait. Celle-ci endossa entièrement l'idée de son mari, d'autant plus qu'elle n'avait pas mis les pieds à Abilène depuis fort longtemps.

C'est ainsi que toute la petite famille monta à bord de la grosse cylindrée pour se diriger résolument vers Abilène. Les appréhensions de Jerry quant à la pertinence d'une telle balade dominicale se révélèrent des plus justes. Le voyage fut tout simplement une catastrophe. repas exécrable nécessitant l'ingurgitation salvatrice d'une bonne dose de Pepto-Bismol. Évidemment, l'épreuve culinaire terminée, nos quatre rescapés du boui-boui durent se résoudre à l'idée de se farcir les 85 kilomètres du chemin du retour.

Complètement lessivés après leur héroïque sortie, les quatre membres de la famille s'écoulèrent littéralement dans le salon de la petite résidence de Coleman. Un lourd silence flottait dans la pièce. Il s'agissait apparemment du calme avant la tempête. Sûrement animé par la volonté de détendre l'atmosphère, Jerry lança: "Ce fut un voyage mémorable, n'est-ce pas? » Il n'en fallut pas plus pour faire sauter le couvercle de la marmite. Les frustrations et les accusations fusèrent de toutes parts. Tous affirmèrent n'avoir jamais voulu aller à Abilène. L'instigateur de la randonnée, le beau-père de Jerry, justifia sa proposition par le fait qu'il croyait sincèrement distraire ses hôtes de leur ennui. L'idée de se rendre à Abilène par une telle chaleur le rebutait, mais il était prêt à se sacrifier pour faire plaisir à sa fille et à son gendre. Si cela n'avait été que de lui, il serait resté à la maison à jouer 
aux dominos! Tous les membres de la famille tinrent le même langage. Ils affirmèrent s'être ralliés à contrecœur à la proposition de la sortie afin de ne pas déplaire aux autres. En fait, les membres de la famille se dirent victimes de la pression que les autres avaient exercée sur eux.

Le paradoxe d'Abilène met en exergue le fait que des personnes raisonnables et sensés puissent, au contact des membres d'un groupe, commettre, de leur propre gré, des actions insensées et contraignantes. En somme, les victimes du Paradoxe d'Abilène font carrément le contraire de ce qu'ils veulent faire.

\section{Précisions définitionnelles}

Les victimes du Paradoxe d'Abilène conservent, au moment de la prise de décision, toute leur pensée critique. Aussi, ces victimes éprouvent un vif malaise (dissonance cognitive) eu égard à la nature de la décision émanant de leurs échanges avec leurs congénères. Ces victimes sont en quelque sorte déchirées par la présence d'une contradiction existant entre leur cognition et leur comportement (dilemme). Motivée par leur besoin de préservation de leurs acquis affectifs et/ou matériels, leur adhésion au choix retenu par le groupe n'est pas volontaire et inconditionnelle, mais contrainte et calculée. Les membres craignent qu'une séparation de leur groupe n'entraîne un étiolement de leurs acquis.

En plus d'être conditionnée par les besoins des membres, l'adhésion contrainte est fortement modulée par la perception naïve qu'entretiennent les membres sur les préférences de leurs collègues. Les victimes du Paradoxe d'Abilène sont persuadées que tous les autres membres du groupe partagent une opinion commune divergente de leur évaluation de la situation (ignorance pluraliste). Craignant d'être perçues par les autres comme des trouble-fête et éventuellement d'être ostracisées par ceux-ci, ces victimes du Paradoxe d'Abilène se comportent comme si elles étaient d'accord avec les propositions avancées par leurs collègues.

Par ailleurs, les membres du groupe, n'affichant qu'un consentement de façade (assujettissement à l'opinion majoritaire ou obligation de plaire aux autres), nourrissent de sérieux doutes à l'endroit des résultats escomptés. Le scepticisme tacite des victi- mes du Paradoxe d'Abilène les confine dans une position de passivité. Prenant leurs distances par rapport aux choix du groupe (détachement décisionnel), ces victimes se contentent d'assumer un rôle de simple exécutant, tout en espérant l'intervention protectrice de la providence.

Lorsque confrontés aux conséquences désastreuses découlant de la prise de décision erronée du groupe, les victimes du Paradoxe d'Abilène expriment ouvertement des émotions négatives (insatisfaction, frustration, colère et conflit interpersonnel).

\section{Le scepticisme tacite des victimes du Paradoxe d'Abilène les confine dans une position de passivité.}

Finalement, le Paradoxe d'Abilène se veut un phénomène consubstantiel à l'expression d'un leadership déficient par absence ou inefficacité.

\section{Applications organisationnelles}

Nous proposons une application du Paradoxe d'Abilène à une entreprise familiale œuvrant dans le secteur de la récréologie forestière, à savoir l'exploitation d'une pourvoirie. Initialement, l'entreprise sélectionnée faisait partie d'un échantillon de cinq autres entreprises familiales n'ayant pu franchir avec succès la phase de lancement, soit une période de 24 mois subséquente à la constitution légale de l'entreprise ${ }^{3}$. Il s'agissait de rédiger six études de cas permettant l'identification des carences d'adaptation managériale au moyen du modèle de l'organisation apprenante élaboré par Argyris ${ }^{4}$. Lors des entrevues avec les propriétaires des entreprises, nous avons été étonnés de constater l'existence de fortes similitudes entre l'expérience vécue par les membres de l'entreprise $n^{\circ} 3$ et les prescriptions du Paradoxe d'Abilène émanant de l'expérience personnelle de Jerry Harvey en 1971. Nous avons donc décidé d'isoler cette entreprise des cinq autres et d'analyser son funeste sort à la lumière du Paradoxe d'Abilène.

\section{Méthodologie}

L'entreprise analysée est une petite entreprise familiale exploitant une pourvoirie offrant à leurs hôtes la 
possibilité de pêcher, de chasser, de faire des randonnés en forêt et de déguster les produits du terroir.

Les propriétaires de l'entreprise familiale sont trois frères et une sœur. Afin de garantir leur anonymat, des noms fictifs seront utilisés. De plus, la région où se sont déroulés les événements ainsi que l'époque seront passées sous silence.

Les quatre propriétaires de l'entreprise ont tous été interviewés individuellement. Le format des entretiens dirigés était le même pour les quatre participants à l'étude. Les entretiens duraient en moyenne $75 \mathrm{mi}$ nutes et s'étalaient sur huit questions ouvertes présentées au tableau 1. Lors de la tenue des entretiens, aucun des participants ne connaissait le phénomène du Paradoxe d'Abilène.

La rencontre avec les participants eut lieu quatorze mois après l'abandon définitif des activités de démar- rage de l'entreprise familiale. Au moment de la cueillette de l'information, l'âge des participants était le suivant : Réal (51 ans), Roland (46 ans), Roxane (42 ans) et Robert (35 ans).

Le choix du type d'instrument de cueillette de l'information s'est arrêté sur l'entretien dirigé. Cet instrument de création d'information s'avère particulièrement approprié pour le traitement de problématiques (prises de décision collective déficientes) ayant préalablement été balisées par un cadre conceptuel (Paradoxe d'Abilène) ${ }^{5}$. Qui plus est, à l'instar de l'entretien non directif, l'entretien dirigé permet la synthèse de données affichant une forte teneur émotionnelle ${ }^{6}$.

Conséquemment, pareille capacité de synthèse se révèle des plus congrues eu égard à l'objet de la présente analyse : un fiasco d'entreprise lors de la phase de lancement.

\section{Tableau 1 - Questions de l'entretien dirigé}

1. Connaissez-vous le phénomène du Paradoxe d'Abilène ?

- Si oui, pouvez-vous le définir?

2. Qu'est-ce qui vous a incité à créer cette entreprise avec les membres de votre famille ?

3. Vous sentiez-vous libre d'adhérer à ce projet ?

- Si non, qu'est-ce qui faisait pression sur vous ?

4. Quels étaient vos sentiments au moment de la prise de décision de vous lancer en affaires ?

5. Quelle était votre évaluation des chances de succès de l'entreprise ?

6. Suite aux difficultés rencontrées et, finalement, à la fin prématurée du projet, décrivez la nature de vos sentiments?

7. Décrivez la nature du leadership présent dans l'entreprise ?

8. Recommenceriez-vous la même aventure ?

- Dites pourquoi ?

\section{Résultats}

\section{Motivation à créer une entreprise}

Après avoir vérifié le niveau de connaissances des participants relativement au Paradoxe d'Abilène, ceux-ci étaient invités à répondre à la deuxième question de l'entretien : qu'est-ce qui vous a incité à créer cette entreprise avec les membres de votre famille ? Il est intéressant de constater que les réponses des quatre participants présentent entre elles une indéniable similarité au chapitre de la peur de la sé- paration (préservation des acquis) et d'une certaine forme d'ignorance pluraliste (croyance erronée à l'effet que les autres ont une position unifiée, diamétralement opposée à la vôtre). Tous les participants ont fait part d'une approche calculée et instrumentale où le retour sur l'investissement exigeait une prise de risque personnelle (peur de la séparation). De plus, les participants estimaient que les autres membres de la famille désiraient s'investir tout autant sinon plus qu'eux dans le projet (variante de l'ignorance pluraliste). Examinons certains extraits significatifs de leurs réponses. 
Réal : « [...] Et puis, par hasard, je suis tombé sur une annonce dans le journal. Terre à vendre ! Il y avait un gars qui voulait vendre une terre de 92 acres. Ça fait pas mal grand. Je pense que c'est là que ça a cliqué. J'en ai parlé au reste de la tribu; la famille. [...] Il y avait tout ce qu'il fallait pour se partir une pourvoirie et attirer les touristes étrangers ou les plaisanciers d'ici. [...] Il n'y en a pas un qui m'ait dit non. Même que Roxane en rajoutait. Elle avait lu dans une revue que ce type d'entreprise-là constituait la voie de l'avenir du tourisme au Québec. Les gens venaient de l'Europe et de l'Asie pour faire des excursions sur le fleuve et observer les baleines. Elle disait que ça marchait très fort. Je vous le dis, ils avaient tous l'air plus emballé que moi du projet. Moi, je voyais ça juste comme un "sideline". Je gardais ma job de "chauffeur" d'autobus et je travaillais là-dessus entre mes "runs" du matin et du soir et les fins de semaine. [...] J'ai lancé une idée, un point c'est tout. Les gens étaient libres de la prendre ou de la laisser. »

Roland : «[...] J'ai suivi, un peu comme un mouton. C'est Réal qui a parti tout ça. Puis après, mon autre frère et ma sœur ont embarqué. À les entendre parler, c'était une mine d'or! L'argent était pour pousser dans les arbres. J'aurais dû rester tranquille et écouter ma femme. [...] Mais si je décidais de ne pas embarquer et que ça marche, je manquais une maudite belle occasion de faire une passe. C'est un peu comme Loto-Québec. Si ton nom n'est pas sur la liste lorsque le gros lot sort, tant pis pour toi !»

Roxane : «Je trouvais l'idée intéressante mais je ne me serais jamais engagée dans ce projet toute seule. [...] Je pensais surtout que ce serait bon pour Robert. L'usine de "plywood" avait fermé ses portes et il se cherchait une job. [...] J'étais bien prête à faire ma part, mais je ne voulais pas quitter mon emploi pour m'investir exclusivement dans cette aventure. [...] J'ai en quelque sorte suivi la parade. Ce sont les gars qui ont poussé pour qu' on fasse le "move" d'acheter le terrain. Il n'y avait pas seulement que Réal qui poussait. Roland et Robert ne donnaient pas leur place. »

Robert: "Ma situation était différente de celle des trois autres. Au moment où on a pris ensemble la décision, j'étais sans travail depuis deux mois environ. Je faisais des petites "jobines" ici et là mais rien de très payant. [...] Je me suis dis : après tout, faire ça ou faire autre chose. [...] Réal et Roxane nous en ont mis plein les yeux. Roland ne parlait pas beaucoup. C'est un gars plutôt silencieux. [...] Réal avait même fait des plans sur des feuilles quadrillés. Il avait divisé son projet en trois phases. [...] Roxane nous avait dit que les gîtes forestiers, ça marchait très fort en Scandinavie. [...] Moi je voyais ça comme un placement. Si ça marche tant mieux ! Sinon, on n'avait seulement qu'à vendre le terrain et à rentrer dans notre argent. »

\section{Nature du consentement}

La troisième question de l'entretien permettait de sonder la nature de l'adhésion des participants au projet. Là encore, tous les participants affichent entre eux une nette ressemblance en matière de consentement au projet de création d'entreprise. Selon leurs réponses, la nature de leur consentement était fortement conditionnée par la pression qu'exerçaient sur eux les autres membres de la fratrie. Les extraits suivants mettent en lumière la présence d'un choix contraint chez les membres de l'entreprise.

Réal : "C'est sûr que j'étais libre de dire oui ou de dire non. Surtout que c'était mon idée. Mais, bien honnêtement, savoir ce que je sais aujourd'hui, je ne me serais jamais embarqué dans cette galère. Vous savez, c'est toujours facile de faire des reproches aux autres une fois que les choses sont faites. [...] Je suis allé de l'avant dans le projet à cause d'eux. Ce sont eux qui m'ont encouragé à continuer et à aller me renseigner auprès du ministère de l'Agriculture, des Pêcheries et de l'Alimentation; auprès de Tourisme Québec; auprès de la Société de la faune et des parcs du Québec. »

Roland: «Je me suis senti pris. Ça s'est fait d'une drôle de façon. Les trois voulaient s'embarquer dans cette patente-là. J'aurais pu refuser et c'est ça que j'aurais dû faire. Mais je me sentais mal à l'aise de ne pas m'impliquer dans une affaire de famille. Les trois auraient roulé ensemble puis moi je serai resté tout seul de mon bord. »

Roxane : «J'étais consciente de ce que je faisais. Je vous mentirais si je vous disais que ce fut une belle expérience. J'ai vécu des choses particulièrement éprouvantes. [...] Je pense que, fondamentalement, j'ai dit oui pour la famille. C'était une façon de nous rassembler et de faire quelque chose ensemble. [...]»

Robert : «Il n’y a personne qui m'ait cassé un bras pour dire oui. [...] Il faut toutefois préciser que j'ai dit 
oui pas uniquement pour me trouver une job. Je me débrouillais avec les petits boulots que j'avais. J'ai dit oui parce que je sentais que ça leur faisait plaisir. Ils voulaient tous, du moins à l'époque, vivre une expérience de retour à la terre. [...] Ouais, je voulais, au plus profond de moi, leur faire plaisir. »

\section{Sentiments au moment de la prise de décision}

L'état émotionnel de tous les participants au moment de la prise de décision était tiraillé, à divers degrés, par une dissonance cognitive, à savoir que le choix personnel des participants était davantage le reflet supputé des préférences des autres que l'expression de leurs appétences intrinsèques. Pareille dissonance cognitive se traduisait par des remises en question et des craintes. Les exemples suivants nous éclairent sur la nature des sentiments des participants.

Réal : " C'est une chose de fantasmer dans un salon. C'est une autre chose de passer à la banque pour faire un emprunt de 25000 \$, et ensuite de passer chez le notaire pour signer les papiers d'achat de la propriété. Je dois vous dire que je "filais" drôle. Je me suis posé la question à savoir si c'était vraiment ça que je voulais faire. [...] Je le faisais-tu pour moi ou pour les autres? Je dois avouer que j'avais des doutes. »

Roland : «J'étais allé trop loin. On était tous rendus chez le notaire. Comme on dit, ce n'est plus le temps de te retenir quand tu as fait dans tes culottes. Je me suis dit: dans quelle affaire, Roland, t'es-tu embarqué ? [...] Je n'ai jamais été confortable avec ma décision. J'aurai dû "fourrer les brakes" chez le notaire et garder mon stylo dans ma poche. »

Roxane : «Vous savez, lorsqu'on était jeunes, on jouait à enlever les pétales d'une marguerite en disant à chaque pétale retirée : "je me marie, je ne me marie pas". J'étais comme ça lorsqu'on a signé les papiers pour s'incorporer et fonder notre entreprise familiale. »

Robert : «Je n'étais ni gai, ni triste. En fait, je ne savais plus au juste ce que je devais faire. Des fois, j'avais le goût de téléphoner au grand et de lui dire que je débarquais. Des fois, je me disais que c'était une occasion en or à ne pas manquer. [...] Financièrement, c'est une aventure qui m'a fait très mal. Mais ce qui est le plus tragique, c'est que maintenant la famille est disloquée. »

\section{Le pronostic des participants relativement aux chances de succès de leur entreprise n'était pas des plus optimistes et ce, même au tout début de l'aventure.}

\section{Évaluation des chances de succès de l'entreprise}

Le pronostic des participants relativement aux chances de succès de leur entreprise n'était pas des plus optimistes et ce, même au tout début de l'aventure. Les extraits des réponses des participants à la cinquième question de l'entrevue reflètent bien la présence généralisée d'un sombre pronostic.

Réal : «Au moment du lancement de l'entreprise, je pensais bien que nos chances étaient bonnes. Si tout le monde avait travaillé ensemble, on aurait augmenté beaucoup nos chances de réussir. Mais, ça n'a pas été long que j'ai commencé à déchanter. Je dirais que six mois après notre visite chez le notaire, j'ai commencé à avoir de sérieux doutes. [...] Une business ne peut pas fonctionner si tu ne t'en occupes pas. Il y en avait seulement deux sur quatre qui travaillaient: moi et Rolland. Les deux autres, Roxane et Robert, se prenaient pour des petits boss. ॥

Roland: «Depuis le tout début, j'ai eu le pressentiment que ça ne marcherait pas. Mais faut croire que je me chassais ça de la tête. [...] Ma femme ne voulait rien savoir du projet. Elle m'avait piqué toute une crise. Encore aujourd'hui, je ne sais pas pourquoi je me suis embarqué là-dedans. [...] J'ai travaillé tant que j'ai pu. [...] Réal a bien des défauts, mais il faut lui donner ça: lui aussi a beaucoup travaillé. Il n'a pas compté ses heures. Robert, ça ne valait pas de la "snoute". Il travaillait seulement quand ça lui disait. [...] Roxane a toujours été forte en gueule. Quand c'est le temps de travailler, zip, elle est disparue. [...] C'était impossible de réussir. »

Roxane: «Moi, ce qui m'a écoeurée dans tout ça, c'est qu'ils ont essayé de nous faire porter le chapeau, à moi et à Robert. Quand tu lances une idée, tu devrais être en mesure de l'assumer. À écouter parler Réal, le projet se faisait facilement en trois étapes, les doigts dans le nez. C'était comme faire un gâteau Duncan Hines. Tu verses le mélange dans le bol. Tu rajoutes du lait, tu brasses et c'est tout. Réal ne nous a jamais dit qu'il y aurait des "mottons" dans la pâte. 
[...] On a acheté en avril et, à l'Action de Grâces, j'ai dit à mon chum que ça ne marcherait pas. »

Robert : «Dès le départ, l'entente n'avait pas d'allure. J'étais le seul à travailler à temps plein sur le projet. Tous les autres avaient un salaire et faisaient ça à temps perdu. Moi, j'étais prêt à en faire autant, mais pas plus que les autres. C'est là que j'ai commencé à diminuer. Je travaillais à temps plein là-dessus et je ne touchais pas une maudite "cenne".

\section{Sentiments au moment des difficultés et de la faillite de l'entreprise}

Les réponses des quatre participants convergent parfaitement quant à leur état émotionnel face au dépérissement de la situation. Tous les participants ont mentionné avoir vécu de l'insatisfaction et des frustrations durant la première année d'exploitation de l'entreprise. Qui plus est, tous les participants ont rapporté avoir éprouvé un sentiment de colère envers les autres membres de la famille. Enfin, un conflit majeur est venu secouer l'association des quatre membres de la fratrie. Certains extraits sont, à cet égard, fort exemplatifs.

Réal : «[...] C'est là que j'ai pété les plombs lorsqu'un soir de février, Robert est venu chez nous pour me demander de racheter sa part. Lui qui s'était poigné le beigne pendant toute l'année ! Là, je lui ai dit ma façon de penser. Je vous le dis que ça "skaquait" dans la maison. [...] Racheter sa part, avec quel argent ! [...] Ce soir-là, j'en ai pleuré une "shot". Peu de temps après, environ deux semaines, ça été le tour de Roxane. Elle voulait elle aussi retirer ses billes de l'entreprise. C'est là qu'on a eu un conseil de famille chez Roland. Seulement les quatre enfants, pas de conjoints! On s'est finalement dit ce qu'on pensait vraiment. »

Roland : «Moi, je ne suis pas un gars qui parle beaucoup. Mais là, j'en avais plein mon casque. [...] Je dois dire que j'étais en maudit après mon frère Réal pour nous avoir placé dans un tel bourbier. J'étais aussi en maudit après les deux autres qui jouaient aux victimes et qui voulaient sacrer leur camp et nous "dumper" toute la merde. Comme solidarité, on repassera ! Ça, je l'aurai toujours sur le cœur. Réal, làdessus, a été correct. Il ne s'est pas poussé lorsque le bateau était en train de couler. [...] Après le fameux meeting du 3 mars, la famille n'existe plus. C'était devenu chacun pour soi. Le lendemain, j'ai téléphoné à Réal et je lui ai dit qu'on avait besoin de monsieur $\mathrm{RE} / \mathrm{MAX} . »$

Roxane : «Moi, si on n'avait laissée tranquille, je ne me serais jamais embarquée là-dedans. [...] Je suis devenue en colère lorsque Réal s'est mis à crier après moi. Là, c'était le bout du bout. C'était celui qui avait eu cette idée lumineuse qui me faisait des reproches. [...] Moi, je voulais débarquer. Je ne voulais pas aller plus loin dans cette connerie. J'avais déjà englouti assez d'argent dans cette aventure. »

Robert: «Ce que je ne comprends pas là-dedans, c'est que si son projet était si bon, pourquoi il ne voulait pas racheter ma part. Je le sais que ce n'est pas correct de penser comme ça, mais j'ai le feeling de m'être fait royalement rouler par ma famille. [...] C'est sûr que Réal est le plus grand responsable, mais les deux autres ne sont pas blancs comme neige. Ils ont tous approuvé son maudit projet de fou. »

\section{Nature du leadership organisationnel}

Les participants partagent la même évaluation de la nature du leadership présent dans l'entreprise familiale. Selon eux, le leadership était carenciel à cause principalement de l'ignorance des membres de l'entreprise. Certains extraits mettent admirablement bien en exergue la présence d'un tel leadership lacunaire en matière de connaissances en récréologie forestière.

Réal : «Je croyais bien faire, mais je n'avais pas de connaissances sur l'industrie touristique. En plus, je n'avais jamais fait de ma vie un plan d'affaires. Tout ce que je connaissais, c'était comment hypothéquer ma maison. [...] Je l'avoue, je ne connaissais rien aux goûts des consommateurs et au service à la clientèle dans une pourvoirie. Il y en a qui se sont servis de ça pour me "blaster". Mais ces gens-là étaient tout aussi ignorants que moi. [...] C'est facile de cracher dans la soupe une fois que tu t'es servi. »

Roland : "On connaissait rien de rien là-dedans. Réal "chauffe" des autobus, Roxane travaille dans un dépanneur puis Robert faisait du "plywood". Moi, j'ai une station-service et je fais de la mécanique générale. Que voulez-vous qu'on fasse dans le bois ? Il n'y en a pas un dans la gang qui est capable de faire la différence entre un sapin et une épinette. On a rêvé debout les yeux grands ouverts. » 
Roxane : "Réal a essayé de prendre le "lead" de la business, mais il ne connaissait rien. Ce n'est pas parce que tu as lu deux ou trois articles sur l'industrie récréotouristique que tu peux te lancer là-dedans et avoir du succès. Personne parmi nous n'avait les connaissances financières et techniques pour se lancer dans une telle aventure. »

Robert: «On a eu tout le monde une belle leçon d'humilité. Quand tu veux jouer les "petits Jos connaissants", la plupart du temps ça te pète en plein dans la face. Ça ne prend pas un gros quotient intellectuel pour comprendre ça. Là-dessus, on a tous été stupides. On a tous voulu jouer à Daniel Boone. »

\section{Intention de recommencer la même aventure}

Aucun des quatre participants n'a exprimé l'intention de recommencer la même aventure. Ils admettent tous avoir commis une erreur, voire une folie. Les extraits suivants soulignent, entre autres, l'amertume et les regrets des membres de l'entreprise.

\section{L'émergence d'un consensus rapide constitue souvent le prodrome d'un profond dysfonctionnement groupal.}

Réal : «Regardez-moi dans les yeux : "never, over my dead body". C'est-tu assez clair ? Ça été une catastrophe ! [...] Semble-t-il qu'on n'est jamais trop vieux pour commettre des erreurs. J'en ai commis toute une ! [...] Là, présentement, je rembourse mes dettes à chaque mois à la banque. Heureusement que je n'ai pas touché à mes RÉER. Faut quand même le faire! Je fais des versements à la banque, avec des intérêts, pour un voyage en enfer! Plus stupide que ça, tu meurs! »

Roland : «Oh que non! Jamais de la vie ! Ça été une erreur de a à z. [...] On n'a jamais eu un "crisse" de client! Par contre, sans trop le savoir, on a créé une entreprise de démolition. On a démoli la famille; on a démoli notre compte de banque; et on a démoli notre réputation. Tout le monde au village est au courant de nos chicanes et de nos dettes. [...] C'est bien spécial, ce que j'ai vécu. Quand tu dis que tes propres frères et ta propre sœur font un détour pour aller "gazer" ailleurs ! [...] Pour moi, personnellement, ça été l'enfer.
Je suis passé à deux doigts d'un divorce à cause de ça. »

Roxane : «Non! Non! Une fois, c'est assez. Je l'avoue, nous avons fait une erreur. L'essentiel, maintenant, c'est de tourner la page et de passer à autre chose. Comme dit la chanson, si je pouvais "rewinder" ma vie, je n'aurais jamais fait ça. J'aurais dû arrêter Réal quand c'était le temps. J'ai voulu aider Robert et ça m'est revenu en pleine face. [...] Aujourd'hui, on ne se parle plus. Je ne sais pas si la famille va reprendre un jour. Peut-être avec le temps ! »

Robert : " Jamais ! Je ne suis pas maso à ce point-là. [...] Des fois, je m'en veux tellement. Ça allait tellement bien avant. Moi, ce que je veux c'est une job, cinq jours par semaine, neuf à cinq. Je n'ai jamais voulu dans la vie devenir millionnaire et travailler comme un débile. Moi, ça ne m'intéresse pas d'être un entrepreneur et d'être obligé de travailler le soir et les fins de semaine. Je veux un salaire et ne pas me "bâdrer" du reste.

\section{Discussion et interprétation}

Les informations recueillies au moyen des entretiens avec les quatre membres de l'entreprise familiale ayant rendu l'âme lors de sa phase de lancement se révèlent particulièrement éclairantes quant aux effets insidieux de l'obtention d'un consentement spontané des membres d'un groupe. L'étude des tribulations des quatre entrepreneurs indique que la détérioration rapide de la viabilité de l'entreprise n'est pas tributaire d'une gestion lacunaire des conflits, mais plutôt d'une gestion carencielle de l'accord. L'émergence d'un consensus rapide constitue souvent le prodrome d'un profond dysfonctionnement groupal. Ayant fait l'économie d'un examen critique et serré des propositions avancées, les membres du groupe participent, à leur insu, à une prise de décision déficiente. Il importe donc pour les dirigeants d'entreprise de se montrer particulièrement circonspects à l'endroit des accords obtenus dans la facilité. Ceux-ci doivent sérieusement se questionner sur la plausibilité d'un choix collectif de qualité obtenu rapidement et facilement.

La gestion fautive de l'accord survenu entre les quatre membres de l'entreprise familiale analysée apparaît découler de la présence de quatre syndromes d'étiolement de la qualité décisionnelle, à savoir : 1) le syndrome de la témérité, 2) le syndrome de l'homogé- 
néité, 3) le syndrome de la futilité et 4) le syndrome de la mutité. L'existence de ces syndromes a d'ailleurs été signalée par certains chercheurs ${ }^{7}$. Examinons la teneur de chacun d'eux.

Le syndrome de la témérité repose sur une absence de peur de l'inconnu. Les gens qui en sont victimes recourent à la banalisation des efforts requis pour atteindre un résultat. Leur tendance à sous-estimer arrogamment les difficultés peut, malheureusement, induire en erreur les gens de leur entourage. Faussement convaincus de la facilité déconcertante de l'entreprise qui leur est proposée, ces gens s'engagent candidement dans des activités excédant de beaucoup leur niveau de compétences. L'expression anglaise « a walk in the park » traduit très bien l'état d'esprit des gens mystifiés par ce syndrome. L'analyse du contenu des réponses des quatre participants à l'étude permet d'identifier Réal, l'aîné de la famille, comme le principal agent inducteur du syndrome de la témérité. Les trois extraits suivants démontrent fort bien l'expression du syndrome de la témérité.

Réal : «Je me suis dis que n'importe quel "smatte" était capable de faire ça. »

Roland : «C'est Réal qui a parti tout ça. Puis après, mon autre frère et ma sœur ont embarqué. À les entendre parler, c'était une mine d'or ! »

Roxane : «À écouter parler Réal, le projet se faisait facilement en trois étapes, les doigts dans le nez. C'était comme faire un gâteau Duncan Hines».

Le deuxième syndrome, celui de l'homogénéité, se résume à la convergence des modes de pensée des membres de l'entreprise. Provenant du même milieu familial, habitant la même région et affichant un degré de scolarité sensiblement analogue - aucun des participants à l'étude n'a complété son secondaire cinq -, les quatre membres de l'entreprise familiale ont été soumis à un processus de socialisation passablement similaire ayant principalement pour effet l'homogénéisation de leurs conceptions (perceptions et cognitions) du monde. Il est bien connu que le manque de diversité culturelle ou paradigmatique a tendance à confiner les individus dans l'adoption de positions rigides et réductionnistes ${ }^{8}$.

Le syndrome de la futilité est très présent dans la problématique investiguée. Il est étonnant de constater que les membres de l'entreprise ont investi collectivement tout près de $200000 \$$ dans un projet tout à fait secondaire. En aucun temps les échanges avec les membres de l'entreprise n'ont permis d'identifier la présence d'une passion certaine et soutenue pour l'exploitation d'une pourvoirie. Pour certains, cette activité était une occasion d'affaires ou une toquade passagère; pour d'autres, elle s'est rapidement révélée une contrainte. Les extraits suivants mettent en lumière l'existence, chez les membres de la famille, du syndrome de la futilité.

Réal : "Moi, je voyais ça juste comme un "sideline". Je gardais ma job de "chauffeur" d'autobus et je travaillais là-dessus entre mes "runs" du matin et du soir et les fins de semaine. [...] Pour moi, c'était une activité secondaire qui, si on travaillait ensemble, pouvait être rentable. [...] Je voyais ça surtout comme un "trip". Une sorte d'évasion dans la nature. Je n'en ai jamais fait une question de vie ou de mort. »

Roland : «Mais, si je décidais de ne pas embarquer et que ça marche, je manquais une maudite belle occasion de faire une passe. C'est un peu comme LotoQuébec. Si ton nom n'est pas sur la liste lorsque le gros lot sort, tant pis pour toi !»

Roxane : " J'étais bien prête à faire ma part, mais je ne voulais pas quitter mon emploi pour m'investir exclusivement dans cette aventure. Il n'était pas question pour moi de quitter le village pour aller vivre dans le fin fond d'un rang. »

Robert : «Je me suis dis : après tout, faire ça ou faire autre chose. [...] Moi, je voyais ça comme un placement. Si ça marche tant mieux! Sinon, on n'avait seulement qu'à vendre le terrain et à rentrer dans notre argent. »

\section{Le syndrome de la témérité repose sur une absence de peur de l'inconnu. Les gens qui en sont victimes recourent à la banalisation des efforts requis pour atteindre un résultat.}

Enfin, le dernier syndrome, celui de la mutité, fait référence à l'existence d'une spirale du silence occultant les désaccords existant entre les membres du groupe. Largement analysée par bon nombre d'au- 
teurs ${ }^{9}$, la spirale du silence est en fait la conséquence de l'ignorance pluraliste, c'est-à-dire la croyance non fondée d'une personne à l'effet que les autres partagent une position commune divergente - en teneur ou en intensité - de la sienne. Afin d'éviter les affres de la marginalisation, la personne, se croyant à tort minoritaire, se campe dans un silence protecteur. Dans le cas qui nous intéresse, il aura fallu tout près d'un an pour que les membres de l'entreprise familiale brisent le cycle de la spirale du silence.

\section{Conclusion}

La présente étude empirique se veut une tentative d'explication de comportements groupaux irrationnels. S'inscrivant dans une perspective d'étude de cas, l'analyse proposée brosse d'intéressantes pistes de réflexion via l'application du concept de Paradoxe d'Abilène. Encore qu'affichant une appréciable tendance à la corroboration des énoncés prescriptifs du Paradoxe d'Abilène, les données de l'étude demeurent fragmentaires et ce, précisément à cause de l'absence d'un devis de recherche comparatif quasi expérimental. Seule la comparaison systématique de plusieurs entreprises, théâtres de fiascos décisionnels et présentant une substantielle variance en matière entre autres de taille, de secteur d'activités, d'accréditation syndicale et de culture organisationnelle, permettrait d'isoler beaucoup plus finement les variables propres au Paradoxe d'Abilène.

\section{Notes et références}

1 Gérard Ouimet est psychologue et politologue, professeur agrégé de psychologie organisationnelle et de management ainsi que directeur des programmes de certificat à l'école des HEC de Montréal.
2 Harvey, J. (1974). «The Abilene Paradox: The Management of Agreement », Organizational Dynamics, vol. 17, p. 63-80; Harvey, J. (1988). The Abilene Paradox and other Meditations on Management, San Diego, CA, Lexington Books.

3 Fleming, Q.J. (2000). Keep the Family Baggage out of the Family Business : Avoiding the Seven Deadly Sins that Destroy Family Businesses, New York, Simon \& Schuster; Hilburt-Davis, J. et W. Jr. Gibb Dyer. (2002). Consulting to Family Businesses, San Francisco, CA, Jossey-Bass/ Pfeiffer; Posa, E.J. (2003). Family Business, Mason, Ohio, South-Western College Pub.

4 Argyris, C. (1999). On Organizational Learning, Malden, MA, Blackwell Publishing.

5 Leary, M.R. (2000). Introduction to Behavioral Research Methods, Boston, MA, Allyn \& Bacon.

6 Pittenger, D.J. (2002). Behavioral Research Design and Analysis, New York, McGraw-Hill.

7 Harvey, M., M.M. Novicevic, M.R. Buckley et J.R.B. Halbesleben (2004), «The Abilene Paradox after Thirty Years : A Global Perspective », Organizational Dynamics, vol. $33, n^{\circ} 2$, p. $215-226$.

Daft, R.L. (2004). The Leadership Experience, Mason, Ohio, Thomson, South-Western.

9 Glynn, C., A. Hayes et J. Shanahan (1997). «Perceived Support for One's Opinions and Willingness to Speak Out: A Meta-Analysis of Survey Studies on the 'Spiral of Silence' », Public Opinion Quarterly, vol. 61, n 3, p. 452463; Sternberg, R. (1998). « Cost and Benefits of Defying the Crowd in Science », Intelligence, vol. 26, n 3, p. 209215; Taylor, G. (1982). "Pluralistic Ignorance and the Spiral of Silence : A Formal Analysis », Public Opinion Quarterly, vol. 46, n³, p. 311-335. 


\section{Publicité}

Maîtrise en gestion de projet 